\title{
Photoacoustic Doppler axial flow measurement of homogenous media using structured illumination
}

Ruiying Zhang, Junjie Yao, Konstantin I. Maslov, Lihong V. Wang

Ruiying Zhang, Junjie Yao, Konstantin I. Maslov, Lihong V. Wang, "Photoacoustic Doppler axial flow measurement of homogenous media using structured illumination," Proc. SPIE 8943, Photons Plus Ultrasound: Imaging and Sensing 2014, 89431U (3 March 2014); doi: 10.1117/12.2038356

SPIE. Event: SPIE BiOS, 2014, San Francisco, California, United States 


\title{
Photoacoustic Doppler axial flow measurement of homogenous media using structured illumination
}

\author{
Ruiying Zhang, Junjie Yao, Konstantin I. Maslov and Lihong V. Wang ${ }^{*}$ \\ Optical Imaging Laboratory, Department of Biomedical Engineering, Washington University in St. \\ Louis, St. Louis, MO 63130
}

\begin{abstract}
We propose time and frequency domain methods for homogenous flow measurement based on the photoacoustic Doppler effect. Excited by spatially modulated laser pulses, the flowing medium induces a Doppler frequency shift in the received photoacoustic signals. The frequency shift is proportional to the component of the flow speed projected onto the acoustic beam axis. These methods do not rely on particle heterogeneity in the medium. A red-ink phantom flowing in a tube immersed in water was used to validate the methods in both frequency and time domains.
\end{abstract}

Keywords: Photoacoustic Doppler effect, homogenous media, structured illumination, Doppler frequency shift, temporal scaling, Doppler flowmetry

\section{INTRODUCTION}

In biomedical imaging, Doppler ultrasound flowmetry, laser Doppler flowmetry, and Doppler optical coherence tomography are useful flow measurement tools in the study of tumor and cardiovascular diseases ${ }^{1-2}$. However, these methods are based on backscattered signals and require particle heterogeneity in the flowing medium. ${ }^{3-6}$ In comparison, photoacoustic imaging, based on optical absorption contrast $\mathrm{t}^{7}$, is not intrinsically limited by the heterogeneity of the flowing medium. A few recent studies have investigated photoacoustic Doppler (PAD) flowmetry of particle-based phantoms and reported traditional PAD signal strength decreases and eventually diminishes as the particle concentration increases. ${ }^{7,8}$ In this paper, we propose flow measurement methods based on the PAD effect in homogeneous media, where the distance between absorptive molecules is on the scale of nanometers.

Different from traditional PAD implementations, which use a temporally modulated $\mathrm{CW}$ or tone-burst laser excitation, our method uses a spatially modulated pulsed laser excitation to temporally modulate the received PA signals. The modulation frequency of the received PA signals is determined by the acoustic transit time between the neighboring pitches of the spatial modulation. Therefore, the flow of the medium changes the acoustic transit time and thus induces a frequency shift in the received PA signals. As in traditional PAD flow measurements, the flow direction determines the sign of the frequency shift.

\section{METHODS AND EXPERIMETNAL SETUP}

In PA flow measurement of a homogeneous medium, a stationary ultrasonic detector receives signals from the excited region of the medium, which is the acoustic source. Fig. 1 is a schematic demonstrating the principle of our flow measurement method. As shown in Fig. 1(a), if both the acoustic source and the medium are stationary, the PA wave from the stationary acoustic source travels to the transducer surface at the speed of sound in the medium $(\sim 1480 \mathrm{~m} / \mathrm{s}$ in water at $20^{\circ} \mathrm{C}$ ). If both the acoustic source and the medium are moving towards the transducer, the received PA signal will be compressed. In the time domain, this compression results in a signal time course scaling, while in the frequency domain, it induced spectrum broadening, as shown in Fig. 1(b). In this study, we explored a time domain method based on temporal scaling and a frequency domain method based on central frequency shift for PAD flowmetry of homogeneous media.

*lhwang@biomed.wust.edu

Photons Plus Ultrasound: Imaging and Sensing 2014, edited by Alexander A. Oraevsky, Lihong V. Wang, Proc. of SPIE Vol. 8943, 89431U · (c) 2014 SPIE · CCC code: 1605-7422/14/\$18 · doi: 10.1117/12.2038356 

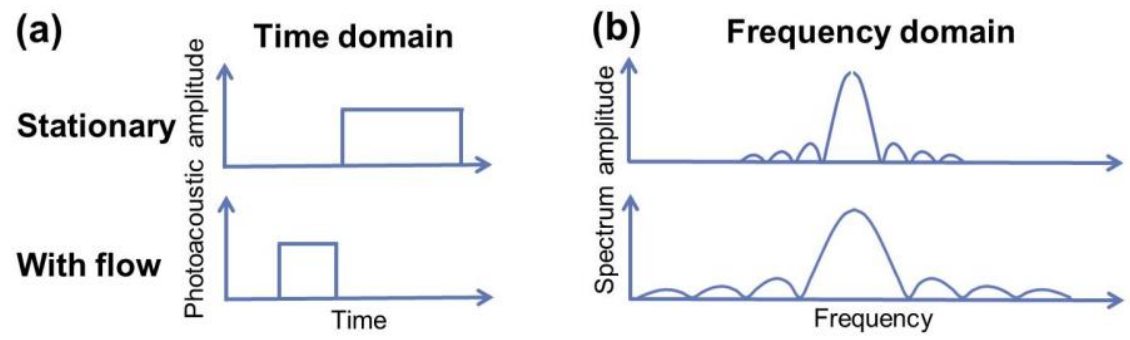

Fig. 1 Schematic of PAD flowmetry of homogeneous media

Temporal Scaling: In the time domain, as demonstrated by Fig. 1(a), the PA signals at different flow speeds will be compressed (or stretched); thus, a temporal scaling factor can be used to calculate flow speeds. The scaling factor that maximizes the product of $p_{1}\left(\varepsilon_{m} t\right)$ and $p_{0}(t)$ can be calculated using Eq. (1), and the flow speed is given by Eq. (2):

$$
\varepsilon_{m}=\underset{\varepsilon}{\operatorname{argmax}} \int_{0}^{t_{\max }} p_{0}(t) p_{1}(\varepsilon t) d t
$$

and

$$
\Delta v_{m}=\left(1-\varepsilon_{m}\right) v_{a}
$$

A temporal scaling factor less than one means the medium is flowing towards the transducer, and vice versa.

Central Frequency Shift: Similar to traditional PAD flow measurement, if the PA signal generated by the moving source has a central frequency with a narrow bandwidth, then the scaling of the spectrum can be represented by its central frequency shift, from which the medium's flow speed and direction can be derived. In our method, the modulation frequency $f_{0}$ can be estimated as

$$
f_{0}=v_{a} / d_{0}
$$

where $v_{a}$ is the speed of sound in the stationary medium, $d_{0}$ is the fringe pitch of the spatially-modulated illumination.

Then, the central frequency shift can be expressed as

$$
f_{P A D}=f_{0} \frac{v_{m}}{v_{a}}
$$

where $v_{m}$ is the flow speed of the medium projected along the acoustic beam axis. A positive frequency shift means the medium is flowing towards the transducer, and vice versa. Note that the PAD frequency shift in Eq. (4) is only half of the traditional ultrasound Doppler shift, due to the one-way emission of the PA signals. In practice, the central frequency of the PA signal can be estimated as the power-weighted mean frequency. ${ }^{9,} 10$

Here, photoacoustic flow sensing based on the PAD effect from a homogeneous medium was demonstrated in a laboratory setup, shown in Fig. 2. A pump (Model 3386, Fisher Scientific and EW-72008-00, Cole Parmer) drove the medium in a clear PVC tube (ClearFlex ${ }^{\mathrm{TM}} 60$ premium, $8 \mathrm{~mm}$ inner diameter) immersed in a water tank. A nanosecond laser of $570 \mathrm{~nm}$ wavelength, focused by a cylindrical lens (300 $\mathrm{mm}$ focal length), was used to generate a 3-mm-long illumination line along the axial direction of the tube. A Ronchi ruling $(50 \mu \mathrm{m}$ pitch size) was placed above the tube, which provided a spatial modulation of the illumination intensity. A diluted red ink solution (optical absorption coefficient: $\left.\mu_{a} \approx 1.2 \mathrm{~cm}^{-1}\right)^{11}$ was used as the flowing medium. The generated photoacoustic waves propagated in the flowing medium and were detected by a flat ultrasonic transducer (V2022 BC, Olympus-NDT). The distance between the illumination spot and the transducer surface was approximately $5.6 \mathrm{~cm}$. The PA signals from the transducer were amplified by $40 \mathrm{~dB}$ and then sampled by an oscilloscope (TDS5034, Tektronix, Inc.). The recorded PA signals were averaged for 256 times. 


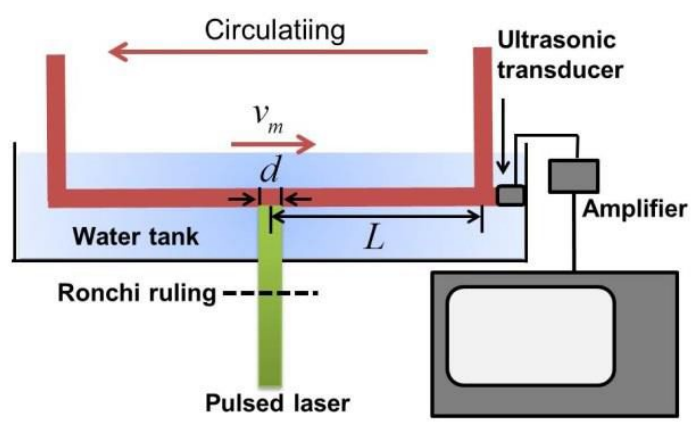

Fig. 2 Schematic of the system setup. The homogenous flow with speed $v_{m}$ is excited by structured illumination, and the generated PA signal is detected by a flat ultrasonic transducer placed in the axial direction of the tube. $L$ is the distance between the illumination spot and the transducer surface, and $d$ is the illumination spot size

\section{RESULTS AND DICUSSIONS}

The Ronchi ruling generated modulated light illumination, which provided a modulated PA signal as shown in Fig. 3 (a). It transformed the otherwise wideband PA signal into a narrowband signal as shown in Fig. 3(b). Without the light modulation, the wideband spectrum would be compressed or stretched as well, depending on the flow direction. However, the narrowband signal facilitated the calculation of absolute flow speeds. The scaling factor can also be used to estimate flow; however, it can detect only relative flow speeds. The total duration of the signal was about $2 \mu$ s, which corresponded to an illumination spot size of $\sim 3 \mathrm{~mm}$. As shown in Fig. 4(a), a faster flow of the medium towards the transducer induces a shorter arrival time of the signal than a slower flow, producing a time shift and a signal time course scaling in the temporal profile of the received PA signal. Correspondingly, Fig. 4(b) shows the frequency spectrum of the PA signal acquired at three different flow speeds. A faster flow of the medium towards the transducer shortens the signal duration and increases the central frequency of the received PA signal, as shown in Fig. 4(b).

(a)
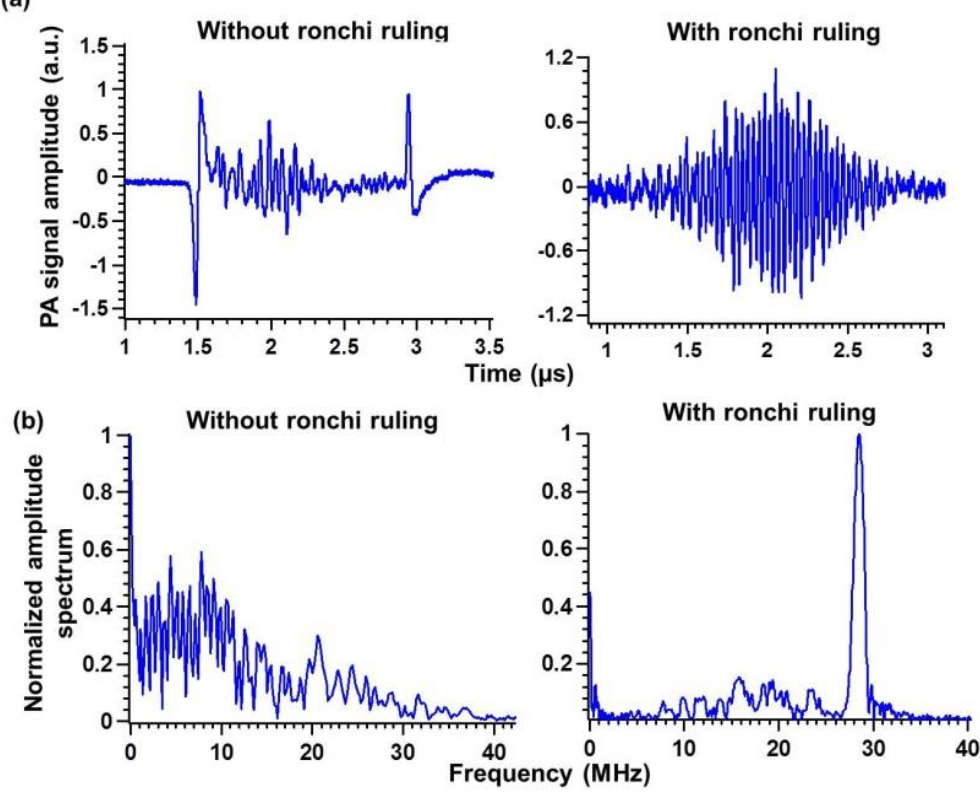

Fig. 3 Representative PA signals in time and frequency domains with and without the modulation of the Ronchi ruling. (a) Representative time-domain PA signals; (b) Representative frequency-domain PA signals. 

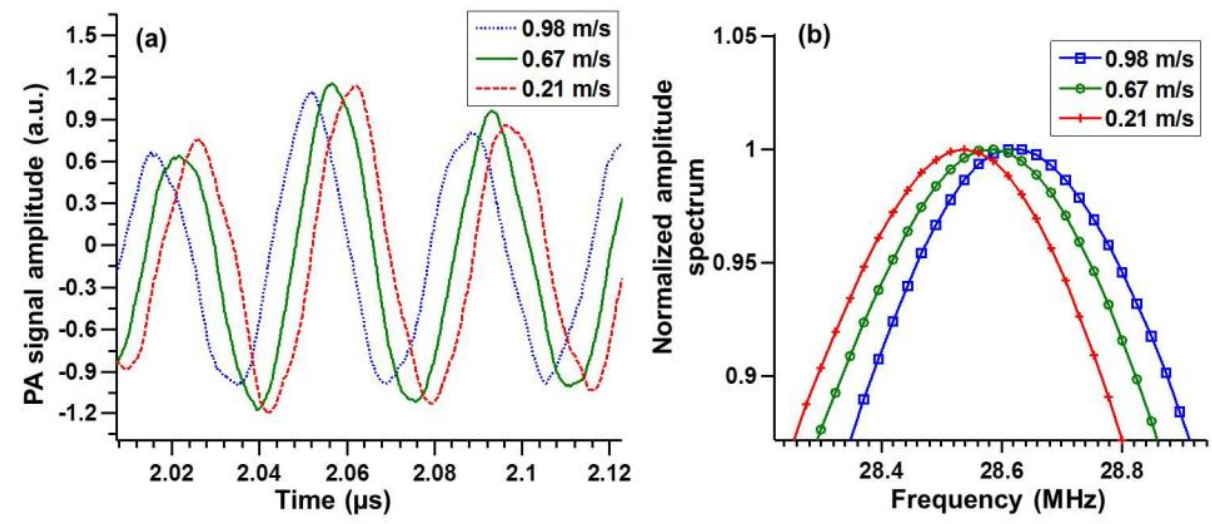

Fig. 4 Modulated PA signals in time and frequency domains at three different flow speeds

The flow speed of the ink solution was varied from $0.020 \mathrm{~m} / \mathrm{s}$ to $1.4 \mathrm{~m} / \mathrm{s}$. The temporal scaling and central frequency shift methods introduced above were used to calculate the flow speeds. It can be seen from Fig. 5(a) and (c), at different flow speeds, the temporal scaling factor and central frequency will vary and based on these changes, and we can calculate the flow speeds. As shown in Fig. 5(b) and (d), the measured flow speeds from the two methods agree well with the preset speeds. The root-mean-square-errors (RMSE) of the two methods were $0.057 \mathrm{~m} / \mathrm{s}$ and $0.089 \mathrm{~m} / \mathrm{s}$, respectively.
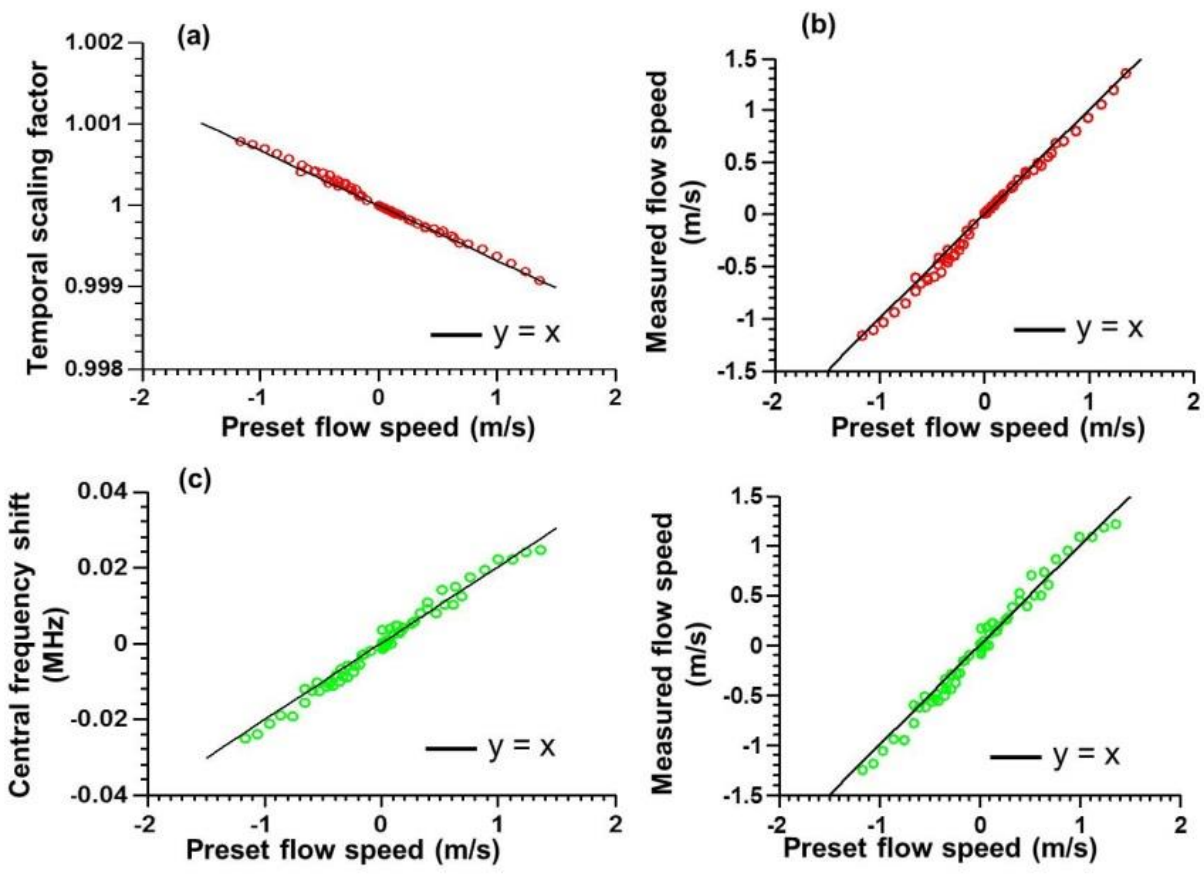

Fig. 5 Flow speed measurement based on temporal scaling and central frequency shift methods.

Based on the scaling property of the Fourier transformation, scaling in the time domain is equivalent to scaling in the frequency domain. Nevertheless, in comparison with the temporal scaling method, the central frequency shift method can sense the absolute flow speed instead of a relative flow speed. Furthermore, the central frequency shift method does not assume uniform flow and homogeneous acoustic property along the sound path between the laser spot and the 
transducer surface. Instead, it requires only uniformity within the illumination spot, which is much easier to satisfy in practice.

It should be noted that our proposed calculation methods assume a uniform flow speed over the depth direction of the tube that the laser can penetrate. However, this is not true for laminar flow, where the center of the tube has the fastest flow. For the absorption coefficient of the ink solution, the 1/e penetration depth of the light was approximately $8.3 \mathrm{~mm}$, larger than the tube diameter. Therefore, the measured flow speed was an average of the actual flow distribution.

In the central frequency shift method, the maximum measurable flow speed is limited by the frequency response of the transducer and SNR. Increasing the bandwidth of the transducer can increase the maximum measurable flow speed. However, since the acoustic signal is attenuated more at higher frequencies, higher frequency components have lower SNR, which will limit the maximum measurable flow speed.

The minimum measurable flow speed, which is the velocity sensitivity of the system, is limited by the signal length and SNR. Increasing the cycle number of the Ronchi ruling can improve the frequency resolution, and thus decrease the minimum measureable flow speed. In addition, zero-padding can be used in the Fourier transformation to improve flow sensitivity. While zero-padding cannot actually improve the frequency resolution, it can smooth the frequency spectrum and thus improve the accuracy of locating the central frequency. ${ }^{12}$

\section{CONCLUSIONS}

We have proposed flow speed measurement methods based on the photoacoustic Doppler effect in a homogeneous medium. Unlike the back-scattering based Doppler method, our methods do not require heterogeneity of the flowing medium on the scale of the spatial resolution. As long as the medium is optically absorptive at a certain wavelength, such as pure water in the infrared region, its flow speed can be measured based on the photoacoustic Doppler effect. While the current experimental setup is more suitable for fast flow $(>2.0 \mathrm{~cm} / \mathrm{s})$ measurement, the slow flow measurement capability can be improved, for example, by increasing the modulation frequency.

\section{ACKNOWLEDGEMENTS}

This research was supported by the National Institutes of Health Grants DP1 EB016986 (NIH Director's Pioneer Award), R01 EB008085, R01 CA134539, U54 CA136398, R01 CA157277, R01 CA159959. L.V.W. has a financial interest in Microphotoacoustics, Inc. and Endra, Inc., which, however, did not support this work. K.M. has a financial interest in Microphotoacoustics, Inc., which, however, did not support this work. The authors would like to thank Ms. Seema Dahlheimer for her close reading of the manuscript. We also appreciate technical assistance from and useful discussions with Lidai Wang, Yong Zhou, Jinyang Liang, Wenxin Xing, Arie Krumholz, and Yan Liu.

\section{REFERENCES}

[1] Nichols, W. W., O'Rourke, M. F. and Vlachopoulos, C., [McDonald's Blood Flow in Arteries: Theoretical, Experimental and Clinical Principles], CRC Press, Boca Raton, p. 1-12 (2001).

[2] Shung, K. K., [Diagnostic Ultrasound: Imaging and Blood Flow Measurements], CRC Press, Boca Raton, p. 1-2 (2005).

[3] White, D. N., "Johann Christian Doppler and his effect--a brief history", Ultrasound Med. Biol. 8(6), 583 (1982).

[4] Evans, D. and McDicken, W., [Doppler ultrasound: physics, instrumentation and signal processing], 2nd ed., Wiley, Chichester, p. 204-207 (2000).

[5] Oberg, P. A., "Laser-Doppler flowmetry", Crit. Rev. Biomed. Eng. 18(2), 125 (1990).

[6] Chen, Z., Zhao, Y., Srinivas, S. M., Nelson, J. S., Prakash, N. and Frostig, R. D., "Optical Doppler tomography", IEEE J. Sel. Topics Quantum Electron. 5(4), 1134 (1999).

[7] Beard, P., "Biomedical photoacoustic imaging", Interface focus 1(4), 602 (2011).

[8] Yao, J., Maslov, K. I., Shi, Y., Taber, L. A. and Wang, L. V., "In vivo photoacoustic imaging of transverse blood flow by using Doppler broadening of bandwidth”, Opt. Lett. 35(9), 1419 (2010). 
[9] Ursem, N. T., Brinkman, H. J., Struijk, P. C., Hop, W. C., Kempski, M. H., Keller, B. B. and Wladimiroff, J. W., "Umbilical artery waveform analysis based on maximum, mean and mode velocity in early human pregnancy", Ultrasound. Med. Biol. 24(1), 1 (1998).

[10] Fernando, K. L., Mathews, V. J. and Clark, E. B., "Mean frequency estimation of narrowband signals", IEEE Signal Process. Lett. 11(2), 175(2004).

[11] van Staveren, H. J., Moes, C. J., van Marle, J., van Gemert, M. J. and Prahl, S. A., "Light scattering in Intralipid$10 \%$ in the wavelength range of 400- $1100 \mathrm{~nm}$ ", Appl. Opt. 30(31), 4507 (1991).

[12] Kay, S. M. and Marple, S. L., "Spectrum analysis - a modern perspective”, Jr., Proc. IEEE 69(11), 1380 (1981). 